\title{
Learning Separable Filters
}

\author{
Amos Sironi*, Bugra Tekin*, Roberto Rigamonti, Vincent Lepetit and Pascal Fua, IEEE Fellow
}

\begin{abstract}
Learning filters to produce sparse image representations in terms of overcomplete dictionaries has emerged as a powerful way to create image features for many different purposes. Unfortunately, these filters are usually both numerous and non-separable, making their use computationally expensive.

In this paper, we show that such filters can be computed as linear combinations of a smaller number of separable ones, thus greatly reducing the computational complexity at no cost in terms of performance. This makes filter learning approaches practical even for large images or 3D volumes, and we show that we significantly outperform state-of-the-art methods on the curvilinear structure extraction task, in terms of both accuracy and speed. Moreover, our approach is general and can be used on generic convolutional filter banks to reduce the complexity of the feature extraction step.
\end{abstract}

Index Terms-Convolutional sparse coding, filter learning, features extraction, separable convolution, segmentation of linear structures, image denoising, convolutional neural networks, tensor decomposition.

\section{INTRODUCTION}

I $\mathrm{T}$ has been shown that representing images as sparse linear combinations of learned filters [31] yields effective approaches to image denoising and object recognition, which outperform those that rely on hand-crafted features [45]. Among these, convolutional formulations have emerged as particularly appropriate to handle whole images, as opposed to independent patches [19], [26], [35], [46]. Unfortunately, because the filters are both numerous and not separable, they tend to be computationally demanding, which has slowed down their acceptance. Their computational cost is even more damaging when dealing with large 3D image stacks, such as those routinely acquired for biomedical purposes.

In this paper, we show that we can preserve the performance of these convolutional approaches while drastically reducing their cost by learning and using separable filters that approximate the non-separable ones. Fig. 1 depicts this behavior in the case of filters designed to classify whether or not a pixel belongs to a blood vessel in retinal scans. Using the learned separable filters is much faster than using either the original non-separable ones or a state-of-the-art implementation of the FFT for all practical filter sizes. We will demonstrate that this is consistently true over a wide range of images.

As we will see, such a result could be achieved by enforcing the separability constraint as part of a convolutional, $\ell_{1}$-based learning framework to directly learn a set of separable filters. However, we have found that an even better result could be obtained by first learning a set of non-separable filters, such as

- A. Sironi, B. Tekin, R. Rigamonti and P. Fua are with the Computer Vision Laboratory, IC Faculty, École Polytechnique Fédérale de Lausanne (EPFL), Lausanne CH-1015, Switzerland.

E-mail: firstname.lastname@epfl.ch

- V. Lepetit is with the Institute for Computer Graphics and Vision, Graz University of Technology, Graz 8010, Austria.

E-mail:lepetit@icg.tugraz.at

This work was supported in part by EU ERC project MicroNano.

* indicates equal contribution.

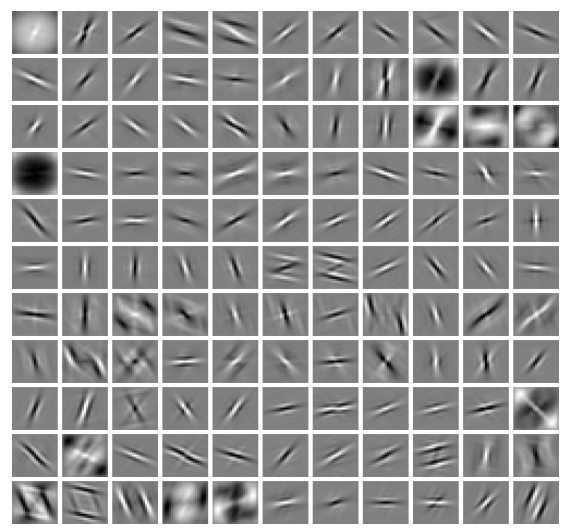

(a)

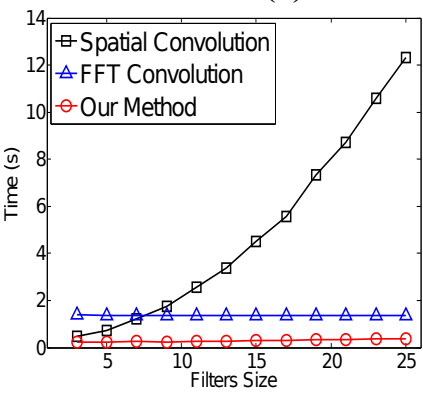

2D images

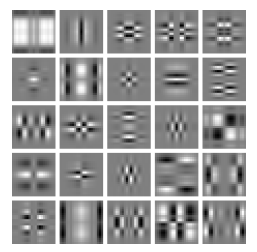

(b)

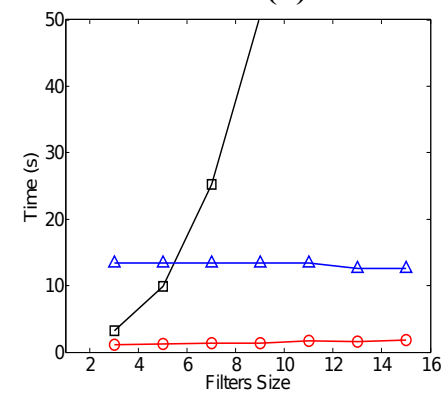

3D image stacks
Fig. 1: Convolutional filter bank (a) learned for the extraction of curvilinear structures in retinal scan images, along with its separable approximation (b). The full-rank filters of (a) can be approximated very precisely as linear combinations of the far fewer separable filters of (b). This allows us to use this property to considerably speed up extraction of learned image features compared with convolutions with the original non-separable filters, even when Fast Fourier Transform is used for both the 2D and the $3 \mathrm{D}$ case, as it is shown by the figures in the bottom row.

those of Fig. 1(a), and then a second smaller set of separable filters, such as those of Fig. 1(b), whose linear combinations can be used to represent the original ones. This makes the approach very efficient because it only requires convolutions 
with a small set of separable filters, which greatly reduces the run-time computational requirements at no loss in accuracy. It also makes the approach very general because it can be applied to any non-separable filter bank, no matter how it has been learned or designed.

We first introduced this idea in [36], where the separable filters are learned by minimizing an objective function that includes low-rank constraints. This approach delivers the desired run-time accuracy and efficiency but at a highcomputational cost during the learning phase. Moreover, the low-rank constraints, being soft constraints, do not guarantee that the resulting filters have rank one. In this situation, separability can be imposed after the fact by truncating the singular values of the filters. Here, we introduce a second approach that explicitly treats the separable filters as products of 1D components, which we learn by tensor decomposition. Our experiments show that this second approach is faster at learning time and more accurate.

In the remainder of the paper, we first discuss related work. We then introduce our approach to separable approximation, first in 2D and then in 3D. Finally, we test our methods on different applications-pixel and voxel classification as well as image denoising and neural networks-and show that the speed-up is systematically significant at no loss in accuracy.

\section{Related work}

Automatic feature learning has long been an important area in Machine Learning and Computer Vision. Neural networks [23], Restricted Boltzmann Machines [18], AutoEncoders [6], Linear Discriminant Analysis [7], and many other techniques have been used to learn features in either supervised or unsupervised ways. Recently, creating an overcomplete dictionary of features-sparse combinations of which can be used to represent images-has emerged as a powerful tool for object recognition [10], [19], [45] and image denoising [13], [27], among others.

However, for most such approaches, run-time feature extraction can be very time-consuming because it involves convolving the image with many non-separable non-sparse filters. It was proposed several years ago to split convolution operations into convergent sums of matrix-valued stages [42]. This principle was exploited in [33] to avoid coarse discretization of the scale and orientation spaces, yielding steerable separable 2D edge-detection kernels. This approach is powerful but restricted to kernels that are decomposable in the suggested manner, which precludes the potentially arbitrary ones that can be found in a learned dictionary or a handcrafted one to suit particular needs. After more than a decade in which the separability property has been either taken for granted or neglected, there is evidence of renewed interest [28], [34]. The scope of those papers is, however, limited in that they are restricted to specific frameworks, while our approach is completely generic. Nonetheless, they prove a growing need for fast feature extraction methods.

Among other recent feature-learning publications, very few have revisited the run-time efficiency issue. The majority of those advocate exploiting the parallel capabilities of modern hardware [14], [30]. However, programming an FPGA unit as in [14] is cumbersome. Exploiting the Graphics Processing Unit as in [30] is an attractive alternative, but the time required for memory transfers between the CPU and the GPU is often prohibitive in practice.

An interesting recent attempt at reducing computational complexity is the approach of [38], which involves learning a filter bank by composing a few atoms from an handcrafted separable dictionary. Our own approach is in the same spirit but is much more general as we also learn the atoms. As shown in the results section, this results in a smaller number of separable filters that are tuned for the task at hand.

[17] learns separable dictionaries in the case of classical sparse coding, i.e., not in a convolution-based approach. The authors show that using separable items as compared to unstructured ones, it is possible to deal with larger images. However the dimensions of the images used are smaller than those typically handled by convolutional sparse coding approaches. Moreover, we will show that directly learning separable filters yields results worse than those of their unstructured counterpart. Here we overcome this limitation by introducing separability at a later stage of the learning process. We first learn a set of non-separable filters and then approximate them as linear combinations of a small set of separable ones, which are specific for the particular application.

Finally, the authors of [9] propose a way to reduce the time it takes to learn non-separable filters. This makes their approach complementary to ours as it could be used to speed up the first step of our algorithm.

\section{Learning 2D Separable Filters}

Most dictionary learning algorithms operate on image patches [10], [27], [31], but convolutional approaches [19], [26], [35], [46] have been recently introduced as a more natural way to process arbitrarily-sized images. They generalize the concept of feature vector to that of feature map, a term borrowed from the Convolutional Neural Network literature [24]. In our work, we consider the convolutional extension of Olshausen and Field's objective function proposed in [35].

Formally, $J$ filters $\left\{\mathbf{f}^{j}\right\}_{1 \leq j \leq J}$ are computed as

$$
\underset{\left\{\mathbf{f}^{j}\right\},\left\{\mathbf{m}_{i}^{j}\right\}}{\operatorname{argmin}} \sum_{i}\left(\left\|\mathbf{x}_{i}-\sum_{j=1}^{J} \mathbf{f}^{j} * \mathbf{m}_{i}^{j}\right\|_{2}^{2}+\lambda_{1} \sum_{j=1}^{J}\left\|\mathbf{m}_{i}^{j}\right\|_{1}\right),
$$

where

- $\mathbf{x}_{i}$ is an input image;

- * denotes the convolution product operator;

- $\left\{\mathbf{m}_{i}^{j}\right\}_{1 \leq j \leq J}$ is the set of feature maps extracted during learning;

- $\lambda_{1}$ is a regularization parameter.

A standard way to solve Eq. (1) is to alternatively optimize over the $\mathbf{m}_{i}^{j}$ representations and the $\mathbf{f}^{j}$ filters. Stochastic gradient descent is used for the latter, while the former is achieved by first taking a step in the direction opposite to 
the $\ell_{2}$-penalized term gradient and then applying the softthresholding operation ${ }^{1}$ on the $\mathbf{m}_{i}^{j}$ s.

While this formulation achieves state-of-the-art results [37], the required run-time convolutions are costly because the resulting filters are not separable. Quantitatively, if $\mathbf{x}_{i} \in$ $\mathbb{R}^{n_{1} \times n_{2}}$ and $\mathbf{f}^{j} \in \mathbb{R}^{d_{1} \times d_{2}}$, extracting the feature maps requires $\mathcal{O}\left(n_{1} \cdot n_{2} \cdot d_{1} \cdot d_{2}\right)$ multiplications and additions. By contrast, if the filters were separable, the computational cost would drop to a more manageable $\mathcal{O}\left(n_{1} \cdot n_{2} \cdot\left(d_{1}+d_{2}\right)\right)$. This cost reduction becomes even more desirable in biomedical applications that require processing large 3D image stacks.

Our goal is therefore to make our filters separable without compromising their descriptive power. One way to do this would be to explicitly write the $\mathbf{f}^{j}$ filters as products of 1D filters and to minimize the objective function of Eq. (1) in terms of their coefficients. Unfortunately, this would involve a quartic objective function in terms of the unknowns in addition to the $\ell_{1}$ norm of the feature maps and, therefore, a very difficult optimization problem.

In the remainder of this section, we introduce two different solutions to overcoming this problem. The first involves a slight modification of the objective function of Eq. (1) to make the learned filters separable by lowering their rank. The second exploits the fact that arbitrary filters of rank $R$ can be expressed as linear combinations of $R$ separable filters [33]. This second solution is more general than the first as it can be applied to any filter bank. It involves approximating the $\mathbf{f}^{j}$ filters of Eq. (1) with linear combinations of separable filters, and we give two methods two obtain these filters. We will show in the Results section that the resulting separable filters, unlike those obtained using the first solution, retain the full discriminative power of the full-rank ones.

\subsection{Penalizing High-Rank Filters}

A straightforward approach to finding low-rank filters is to add a penalty term to the objective function of Eq. (1) and to solve

$$
\begin{aligned}
& \underset{\left\{\mathbf{s}^{j}\right\},\left\{\mathbf{m}_{i}^{j}\right\}}{\operatorname{argmin}} \sum_{i}\left(\left\|\mathbf{x}_{i}-\sum_{j=1}^{J} \mathbf{s}^{j} * \mathbf{m}_{i}^{j}\right\|_{2}^{2}+\Gamma_{\mathbf{m}, \mathbf{s}}^{i}\right), \\
& \text { with } \Gamma_{\mathbf{m}, \mathbf{s}}^{i}=\lambda_{1} \sum_{j=1}^{J}\left\|\mathbf{m}_{i}^{j}\right\|_{1}+\lambda_{*} \sum_{j=1}^{J}\left\|\mathbf{s}^{j}\right\|_{*},
\end{aligned}
$$

where the $\mathbf{s}^{j}$ s are the learned linear filters, $\|\cdot\|_{*}$ is the nuclear norm, and $\lambda_{*}$ is an additional regularization parameter. The nuclear norm of a matrix is the sum of its singular values and is a convex relaxation of the rank [15]. Thus, forcing the nuclear norm to be small amounts to lowering the rank of the filters. Experimentally, for sufficiently high values of $\lambda_{*}$, the $\mathbf{s}^{j}$ filters become effectively rank 1 and they can be written as products of 1D filters.

Solving Eq. (2), which has the nuclear norm of the filters as an additional term compared to Eq. (1), requires minimal extra effort. After taking a step in the direction opposite of that of

1. Soft-thresholding is the proximal operator for the $\ell_{1}$ penalty term [3]; its

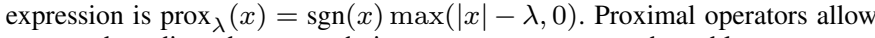
to extend gradient descent techniques to some nonsmooth problems. the gradient of the filters, as described in the previous section, we just have to apply the proximal operator of the nuclear norm to the filters. This amounts to performing a Singular Value Decomposition (SVD) $\mathbf{s}=\mathbf{U D V}^{\top}$ on each filter $\mathbf{s}$, soft-thresholding the values of the diagonal matrix $\mathbf{D}$ to obtain a new matrix $\widehat{\mathbf{D}}$, and replacing $\mathbf{s}$ by $\mathbf{U} \widehat{\mathbf{D}} \mathbf{V}^{\top}$. At convergence, to make sure we obtain separable filters, we apply a similar SVD-based operation but set to 0 all the singular values but the largest one. In practice, the second largest singular value is already almost zero even before clipping.

Choosing appropriate values for the optimization parameters, the gradient step size, $\lambda_{1}$, and $\lambda_{*}$, is challenging because they express contrasting needs. We have found it effective to start with a low value of $\lambda_{*}$, solve the system, and then progressively increase it until the filter ranks are close to one.

\subsection{Linear Combinations of Separable Filters}

We will show in the Results section that the separable filters obtained using the method discussed above give less accurate classification results than the non-separable ones. This is probably because the additional constraints imposed on the filters make the problem too hard to solve optimally, as observed in [17] when learning separable dictionaries for denoising purposes.

We therefore introduce a second approach that exploits the fact that a filter $\mathbf{f}^{j}$ of rank $R$ can always be written as

$$
\mathbf{f}^{j}=\sum_{k=1}^{R} w_{j}^{k} \mathbf{s}^{j, k},
$$

where the $\mathbf{s}^{j, k}$ filters are separable, or equivalently of rank one, and the $w_{j}^{k}$ are scalar weights.

In the $2 \mathrm{D}$ case, such a representation could be obtained by SVD decomposition of each one of the $J \mathbf{f}^{j}$ filters of Eq. (1) independently. However, this would yield a different bank of filters for each $\mathbf{f}^{j}$ and would be inefficient at run-time. To avoid this, we impose that each $\mathbf{f}^{j}$ be written as

$$
\mathbf{f}^{j}=\sum_{k=1}^{K} w_{j}^{k} \mathbf{s}^{k},
$$

where the separable filters $\mathbf{s}^{k}$ are shared among all the nonseparable ones and only the coefficients $w_{j}^{k}$ s change. In this way, convolving the image with all the $\mathbf{f}^{j}$ 's at runtime amounts to convolving it with the separable $\mathbf{s}^{k}$ filters and then linearly combining the results, without any further convolutions.

\subsubsection{Minimizing the Nuclear Norm}

A most direct way to learn the separable filters $\mathbf{s}^{k}$ and scalar coefficients $w_{j}^{k}$ of Eq. 5 would be to solve

$$
\underset{\substack{\left\{\mathbf{m}_{i}^{j}\right\} \\\left\{\mathbf{s}^{k}\right\},\left\{w_{k}^{j}\right\}}}{\operatorname{argmin}} \sum_{i}\left(\left\|\mathbf{x}_{i}-\sum_{j=1}^{J}\left(\sum_{k=1}^{K} w_{j}^{k} \mathbf{s}^{k}\right) * \mathbf{m}_{i}^{j}\right\|_{2}^{2}+\Gamma_{\mathbf{m}, \mathbf{s}}^{i}\right),
$$

where $\Gamma_{\mathbf{m}, \mathbf{s}}^{i}$ is defined in Eq. (3) and includes the nuclear norm. This is a reformulation of Eq. (2) with $w_{j}^{k}=1$ if $j=$ 


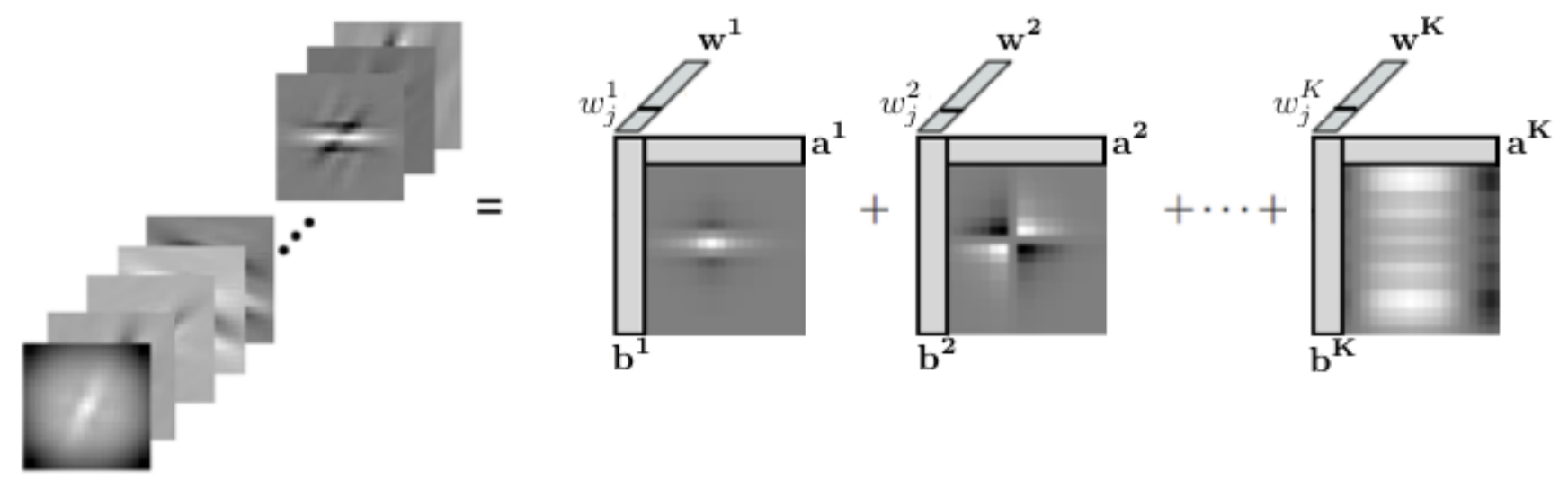

Fig. 2: Tensor decomposition for learning separable filters. Left: A bank of two-dimensional filters is stacked together to form a 3-dimensional tensor, Right: The tensor is decomposed in the sum of $K$ rank-one tensors. Thus, the original filters are approximated by the weighted sum of the separable filters $\mathbf{s}_{k}=\mathbf{a}_{k} \circ \mathbf{b}_{k}$.

$k$ and 0 otherwise. Unfortunately, this objective function is difficult to optimize because its first term contains products of three unknowns, in addition to the second term involving the $\ell_{1}$ norm of the feature maps and the nuclear norm of the filters.

A standard way to handle this difficulty is to introduce auxiliary unknowns, making the formulation linear by introducing additional parameters. Parameter tuning is, however, already difficult in the formulation of Eq. (1), and this would therefore only worsen the situation. We tried instead a simpler approach, which has yielded better results by decoupling the computation of the non-separable filters from that of the separable ones. We first learn a set of non-separable filters $\left\{\mathbf{f}^{j}\right\}_{j}$ by optimizing the original objective function of Eq. (1). We then look for separable filters whose linear combinations approximate the $\mathbf{f}^{j}$ filters by solving

$$
\underset{\left\{\mathbf{s}^{k}\right\},\left\{w_{k}^{j}\right\}}{\operatorname{argmin}} \sum_{j}\left\|\mathbf{f}^{j}-\sum_{k=1}^{K} w_{j}^{k} \mathbf{s}^{k}\right\|_{2}^{2}+\lambda_{*} \sum_{k=1}^{K}\left\|\mathbf{s}^{k}\right\|_{*} .
$$

Even though this may seem suboptimal when compared to the global optimization scheme of Eq. (6), it gives superior results in practice because the optimization process is split into two easier tasks and depends on just two parameters, easing their scheduling.

To solve Eq. (7), we alternately optimize on the filters $\mathbf{s}^{k}$ and on the weights $w_{j}^{k}$ using gradient descent. At each iteration, after moving in the gradient direction, we apply the proximal operator of the nuclear norm to the filters, as described in Section 3.1 to minimize the criterion of Eq. (2).

\subsection{Tensor Decomposition}

The method described in the previous section produces a small set of separable filters that approximate the original ones. However, it requires introducing an additional regularization parameter $\lambda_{*}$ that can be difficult to tune. Moreover, we have experimentally found that its convergence rate is slow, especially when trying to approximate high-rank filters. We therefore introduce here an alternative approach to finding the separable filters $\mathbf{s}^{k}$ and weights $w_{j}^{k}$ of Eq. (5), that relies on tensor decomposition.
Low rank tensor decomposition techniques have been used in many Computer Vision applications [20], [5], [4], [40] to obtain a speed up for a particular application. In this section, we show how tensor decomposition can be used in a general framework to obtain the decomposition of Eq. (4) for an arbitrary filter bank and speed up convolutions. As we will show in Section 5, not only this approach is faster than optimizing Eq. (7), but also returns more accurate approximations.

We start by stacking the $J$ filters $\mathbf{f}^{j} \in \mathbb{R}^{d_{1} \times d_{2}}$ into a 3dimensional tensor $\mathcal{F} \in \mathbb{R}^{d_{1} \times d_{2} \times J}$, where the $j$-th slice of $\mathcal{F}$ corresponds to the $j$-th filter $\mathbf{f}^{j}$, as shown in Fig. 2.

Writing the slices of $\mathcal{F}$ as linear combinations of rankone matrices is equivalent to writing the tensor $\mathcal{F}$ as a linear combination of rank-one tensors

$$
\mathcal{F}=\sum_{k=1}^{K} \mathbf{a}^{k} \circ \mathbf{b}^{k} \circ \mathbf{w}^{k},
$$

where $\mathbf{a}^{k}$ is a vector of length $d_{1}, \mathbf{b}^{k}$ a vector of length $d_{2}$ and $\mathrm{w}^{k}$ a vector of length $J$. The symbol $\circ$ corresponds to the tensor product, that for vectors is also referred to as outer product. Such a decomposition is called Canonical Polyadic Decomposition (CPD) [21] of the tensor $\mathcal{F}$ and the right-hand side of Eq. (8) is called Kruskal form of the tensor. We will refer to $K$ as the rank of the Kruskal tensor.

If tensor $\mathcal{F}$ can be written in the form of Eq. (8), we obtain

$$
\mathbf{f}^{j}=\sum_{k=1}^{K} w_{j}^{k} \mathbf{s}^{k}, \quad \forall j,
$$

where the separable filters are given by the $\mathbf{a}^{k}$ and $\mathbf{b}^{k}$ components of the CPD, that is, $\mathbf{s}^{k}=\mathbf{a}^{k} \circ \mathbf{b}^{k}$. The coefficients $w_{j}^{k}$ necessary to reconstruct the filter $j$ are given by the $j$-th component of the $\mathbf{w}^{k}$ vectors, as shown in Fig. 2.

In general, for a given $K$, we have no guarantee that the decomposition of Eq. (8) exists. Thus, we will compute the best approximation of this form by optimizing

$$
\min _{\left\{\mathbf{a}^{k}, \mathbf{b}^{k}, \mathbf{w}^{k}\right\}_{k}}\left\|\mathcal{F}-\sum_{k=1}^{K} \mathbf{a}^{k} \circ \mathbf{b}^{k} \circ \mathbf{w}^{k}\right\|_{2}^{2} .
$$




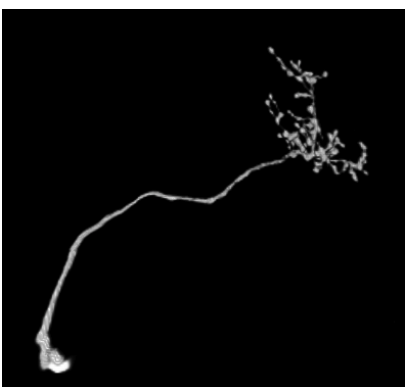

(a)

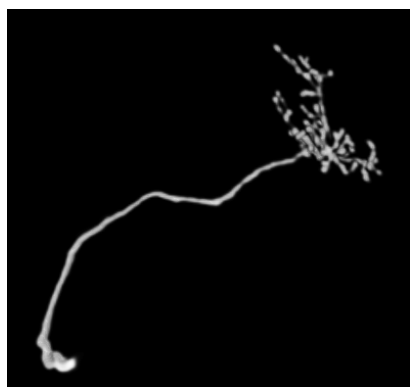

(b)

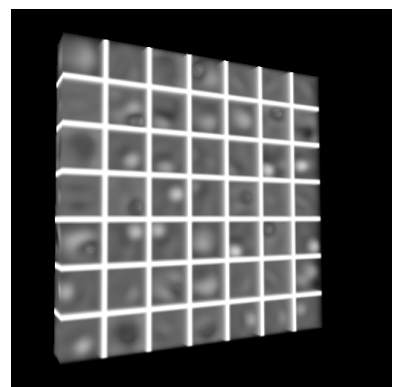

(c)

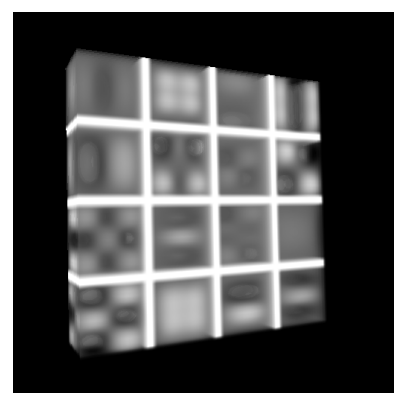

(d)

Fig. 3: Examples of 3D filter banks learned on the OPF dataset [2]. (a) A 3D test image stack. (b) Response of the classifier trained on the separable filter bank output (d). (c) Non-separable filter bank learned by optimizing Eq. (1). (d) The separable filter bank learned by optimizing Eq. (7).

To this end, we use the CP-OPT algorithm of [1], implemented in the MATLAB tensor toolbox, in which Eq. (9) is solved by conjugate gradient descent. Details about the optimization procedure are given in the Appendix.

The rank $K$ of the decomposition is the only parameter of the method. It determines the number of separable filters used to approximate the original filter bank.

In Section 5 we will show that the two strategies based on the linear combination of separable filters, presented in Section 3.2, yields the best results and that they are really close in terms of accuracy and efficiency. In Section 5.6 we will show that the Tensor Decomposition approach of Eq. (9) has faster convergence and gives a better approximation of the original filter bank, compared to the approach of Eq. (7) and therefore should be preferred in practice.

\section{LeARning $N$-D SePARABle Filters}

The computational complexity of feature extraction becomes even more daunting when handling multidimensional data, such as the 3D volume in Fig. 3. Fortunately, our approach to learning separable filters generalizes naturally to any dimension.

As will be shown in the Results section, the formalism of Sections 3.2.1 and 3.3 yields the best results in the $2 \mathrm{D}$ case and we therefore rely on it for the proposed extension.

Minimizing the Nuclear Norm: The structure of the optimization scheme of Eq. (7) of Section 3.2.1 is unchanged. It simply involves a CPD of the filters instead of the SVD decomposition used for the $2 \mathrm{D}$ filters, during the optimization procedure.

Tensor Decomposition: Similarly, the generalization of the method of Section 3.3 to $N$-D filters is straightforward. Let $\left\{\mathbf{f}^{j}\right\}_{j=1}^{J}$ be a set of filters, with $\mathbf{f}^{j} \in \mathbb{R}^{d_{1} \times \cdots \times d_{N}} \forall j$. Let $\mathcal{F}$ be the $(N+1)$-D tensor formed by stacking the $\mathbf{f}^{j}$ 's along the $(N+1)$-th dimension, that is $\mathcal{F}_{i_{1}, i_{2}, \ldots, i_{N}, j}=f_{i_{1}, i_{2}, \ldots, i_{N}}^{j}$. Applying CPD of rank $K$ to $\mathcal{F}$, yields

$$
\mathcal{F} \approx \sum_{k=1}^{K} \mathbf{a}^{k, 1} \circ \mathbf{a}^{k, 2} \circ \cdots \mathbf{a}^{k, N} \circ \mathbf{w}^{k}
$$

Therefore, for all $j=1, \ldots, J, \mathbf{f}^{j} \approx \sum_{k=1}^{K} w_{j}^{k} \mathbf{s}^{k}$, with $\mathbf{s}^{k}=$ $\mathbf{a}^{k, 1} \circ \mathbf{a}^{k, 2} \circ \cdots \mathbf{a}^{k, N}$.

In Section 5.6 we compare the tensor decomposition approach against the approach of Eq. (7), showing that the first one converges faster and gives lower approximations errors.

\section{Results And Discussion}

In this section, we compare the performance and computational complexity that results from using either separable filters or non-separable ones and different strategies for deriving them.

We first introduce these strategies and provide a theoretical analysis of their respective computational complexities. We then test them for three very different purposes:

- classifying pixels and voxels in biomedical images as belonging to linear structures or not;

- denoising;

- performing handwritten digit recognition and drone detection using convolutional neural networks.

We will show that our separable filters systematically deliver a very substantial speed-up at no loss in performance in line with our theoretical analysis. The code and parameters for all these experiments are publicly available at http://cvlab.epfl.ch/software/filter-learning.

\subsection{Competing Strategies}

In the following, we will refer to the non-separable filters obtained by minimizing the objective function of Eq. (1) as $N O N-S E P$ and the separable ones learned using the technique of Section 3.1 as SEP-DIRECT. These essentially constitute baselines that reflect the current state-of-the-art. To provide additional baselines, we also compute separable SEP-SVD and $S E P-C P D$ filters by approximating each $N O N-S E P$ filter by the outer product of its first left singular vector with its first right singular vector computed using SVD in 2D and by rank-1 CPD in 3D, which is the simplest way to approximate a nonseparable filter by a separable one. For completeness' sake, we reimplemented $N O N-S E P$ using the Fast Fourier Transform to perform the convolutions. This approach is known to speed-up convolutions for large enough filters and we will refer to it as NON-SEP-FFT. 
$S E P-C O M B$ and SEP-TD will denote the separable ones we advocate in this paper and whose linear combinations can be used to approximate the non-separable NON-SEP filters as described in Section 3.2. More specifically, SEP-COMB will refer to those that have been learned by minimizing the nuclear norm, as described in Section 3.2.1 and SEP-TD to those obtained by tensor decomposition, as discussed in Section 3.3.

Finally, although the SEP-COMB and SEP-TD filters can be used to write the non-separable ones as linear combinations of them, explicitly computing the coefficients of these combinations is not always necessary. For example, when the filters' output is to be fed to a linear classifier for classification purposes, this classifier can be trained directly on the separable-filters' output instead of that of the non-separable ones. This approach, which we will refer to as $S E P-C O M B^{*}$ and $S E P-T D^{*}$, further simplifies the run-time computations because the linear combinations' coefficients are then learned implicitly at training-time.

\subsection{Computational Complexity}

The different methods described in the previous section are summarized in Table 1 . Here, we provide an analysis of their computational complexities in terms of the number of multiplications required to perform the necessary run-time convolutions.

\subsubsection{The $2 D$ Case}

Let $\mathbf{x} \in \mathbb{R}^{n_{1} \times n_{2}}$ be an image we want to convolve with a filter bank $\left\{\mathbf{f}^{j}\right\}_{j=1}^{J}$, with $\mathbf{f}^{j} \in \mathbb{R}^{d_{1} \times d_{2}}$.

In the NON-SEP case, $J$ convolutions are computed in the spatial domain and each one requires $n_{1} \cdot n_{2} \cdot d_{1} \cdot d_{2}$ multiplications, for a total of

$$
N O N-S E P_{n o p}=J \cdot n_{1} \cdot n_{2} \cdot d_{1} \cdot d_{2}
$$

multiplications.

In the NON-SEP-FFT case, the convolutions are performed in the frequency domain, which involves the following steps:

- Padding $\mathbf{x}$ and $\mathbf{f}^{j}$ with zeros to have the same size $m_{1} \times$ $m_{2}$, where $m_{i}$ is the closest power of 2 larger than $\left(n_{i}+\right.$ $d_{i}-1$ ), for $i=1,2$;

- Computing real-to-complex FFT on the padded image and the $J$ filters;

- Multiplying the resulting discrete Fourier transform (DFT) of the image by that of each filter;

- Computing complex-to-real Inverse FFT (IFFT) on the results.

To decrease the total computational cost of the convolutions, we can precompute the FFT of the filters, at the cost of using more memory.

Assuming that each FFT and IFFT requires $c \cdot m_{1} \cdot m_{2}$. $\log _{2}\left(m_{1} \cdot m_{2}\right)$ complex multiplications where $c$ depends on the specific FFT algorithm being used and that each complex multiplication requires 3 real multiplications, this yields a total of

$$
\begin{aligned}
\text { NON-SEP-FFT } & =3 \cdot c \cdot m_{1} \cdot m_{2} \cdot \log _{2}\left(m_{1} \cdot m_{2}\right) \\
& +3 \cdot J \cdot m_{1} \cdot m_{2} \\
& +3 \cdot J \cdot c \cdot m_{1} \cdot m_{2} \log _{2}\left(m_{1} \cdot m_{2}\right)(11)
\end{aligned}
$$

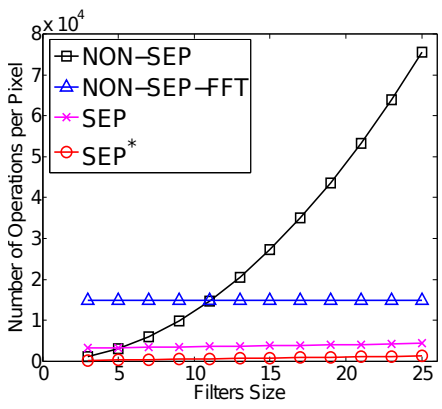

(a)

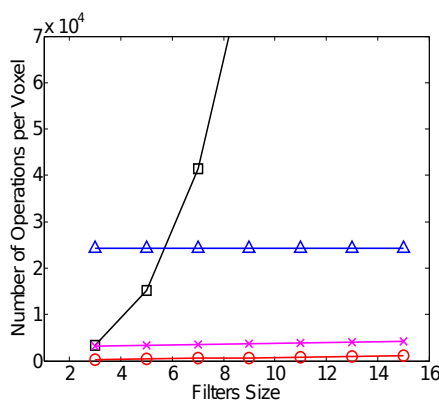

(b)
Fig. 4: Number of operations per pixel to compute convolutions, as a function of the filters size. (a) An image of $488 \times 488$ pixels is convolved with a filter bank of $J=121$ non-separable filters and $K=25$ separable ones. (b) A volume of $114 \times 114 \times 50$ voxels is convolved with a filter bank of $J=121$ non-separable filters and $K=25$ separable ones. The theoretical values are very similar to the experimental time shown in Fig. 1 and they show that the number of operations needed to compute the convolutions with our approach is smaller than both spatial convolution and FFT based convolution.

multiplications. In practice, the value used for the constant $c$ is 2 .

When the filters are separable, the cost of a spatial convolution reduces to $n_{1} \cdot n_{2} \cdot\left(d_{1}+d_{2}\right)$. If the filter bank is composed of $K$ filters, this represents

$$
\mathrm{SEP}^{*}{ }_{n o p}=K \cdot\left(n_{1} \cdot n_{2} \cdot\left(d_{1}+d_{2}\right)\right)
$$

multiplications. This is the total cost for $S E P-C O M B^{*}$ and $S E P-T D^{*}$. In the cases of $S E P-C O M B$ and $S E P-T D$, one must account for the additional cost of linearly combining the results to approximate the $J$ non-separable filters. This requires $n_{1}$. $n_{2} \cdot K \cdot J$ more multiplications, for a total of

$$
\mathrm{SEP}_{n o p}=K \cdot n_{1} \cdot n_{2} \cdot\left(J+d_{1}+d_{2}\right)
$$

multiplications.

In Fig. 4(a), we plot the values of $N O N-S E P_{n o p}$, $N O N-S E P-F F T_{n o p}, \mathrm{SEP}^{*}{ }_{n o p}$, and $\mathrm{SEP}_{n o p}$, normalized by the number of pixels in the image, as a function of the size $d=d_{1}=d_{2}$ of the filters between [3,25]. A 2D test image of size $488 \times 488$ is considered and convolved with $J=121$ non-separable filters and $K=25$ separable ones. Notice that the size of the image is chosen so that the size considered to compute the FFT is a power of 2 for the maximum value of the filters $d=25$. In this way the zero-padding required is minimal, which is at the advantage of the FFT based approach.

Note that these theoretical curves are very similar to those observed experimentally, shown in Fig. 1.

Our code relies on the MATLAB conv2 function for spatial 2D convolutions and on the fftw library for the frequency domain convolutions. Observe that these functions can be run in parallel to further reduce the cost of the convolutions, as shown in Fig. 5.

\subsubsection{The $N-D$ Case}

The generalization to any dimension is straightforward. If $\mathbf{x} \in \mathbb{R}^{n_{i} \times, \ldots, \times n_{N}}$ is an $N$-dimensional image and $\mathbf{f}^{j} \in$ $\mathbb{R}^{d_{1} \times, \ldots, \times d_{N}}$ an $N$-dimensional filter, the cost of a nonseparable convolution becomes $\prod_{i=1}^{N} n_{1} \cdot d_{i}$. The cost of a 
TABLE 1: Summary of the different methods used for our experiments, as described in Section 5.3.

\begin{tabular}{lll}
\hline Method Name & Filter Bank & Run Time Computations \\
\hline$N O N-S E P$ & Non-separable filters learned from Eq. (1) & Spatial convolutions \\
$N O N-S E P-F F T$ & Non-separable filters learned from Eq. (1) & FFT convolutions \\
$S E P-S V D$ & Approximation of $N O N-S E P$ by truncated SVD in 2D & Separable convolutions \\
$S E P-C P D$ & Approximation of $N O N-S E P$ by rank-one CPD in 3D & Separable convolutions \\
$S E P-D I R E C T$ & Separable filters learned from Eq. (2) & Separable convolutions \\
$S E P-C O M B$ & Separable filters learned from Eq. (7) & Separable convolutions + linear combinations \\
$S E P-T D$ & Separable filters learned from Eq. (9) & Separable convolutions + linear combinations \\
$S E P-C O M B^{*}$ and $S E P-T D^{*}$ & As $S E P-C O M B$ and $S E P-T D$ & Separable convolutions \\
\hline
\end{tabular}

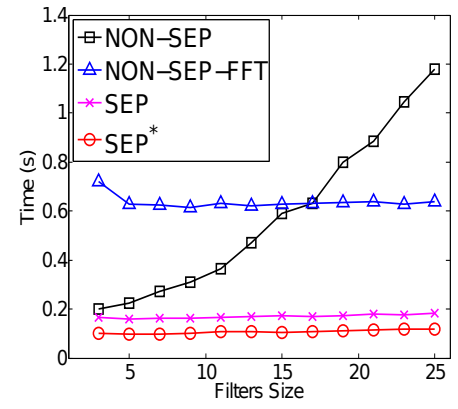

(a)

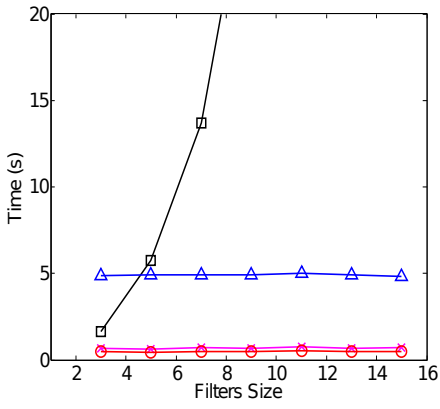

(b)
Fig. 5: Time needed to compute convolutions using a multi-thread MATLAB implementation, as a function of the filters size. (a) An image of $488 \times 488$ pixels is convolved with a filter bank of $J=121$ non-separable filters and $K=25$ separable ones. (b) A volume of $114 \times 114 \times 50$ voxels is convolved with a filter bank of $J=$ 121 non-separable filters and $K=25$ separable ones. Using parallel computation the time needed to compute the convolution is further reduced and our methods are still the most efficient ones. The times are averaged over 5 repetitions.

separable convolution is $\left(\prod_{i=1}^{N} n_{i}\right) \cdot\left(\sum_{i=1}^{N} d_{i}\right)$ and the cost of a FFT is $c \cdot m \cdot \log _{2}(m)$, where $m$ is the product of the closest larger powers of 2 of $n_{i}+d_{i}-1$.

Figs. 4(b) and 5(b) illustrate that using separable filters is even more advantageous for the $3 \mathrm{D}$ case. Here the size of the filters is between 3 and 15 and a $114 \times 114 \times 50$ volume is considered. Again the size of the volume is taken at the advantage of the FFT based approach.

\subsection{Detection of Curvilinear Structures}

Biomedical image processing is a particularly promising field of application for Computer Vision techniques as it involves large numbers of 2D images and 3D image stacks of ever growing size, while imposing strict requirements on the quality and the efficiency of the processing techniques. Here, we demonstrate the power of our separable filters for the purpose of identifying curvilinear structures, a long-standing Computer Vision problem that still remains wide-open when the image data is noisy.

Over the years, models of increasing complexity and effectiveness have been proposed, and attention has recently turned to Machine Learning techniques. In [16], [39] a Support Vector Machine classifier is applied to the responses of ad hoc filters. In particular, [39] considers the Hessian's eigenvalues while [16] relies on steerable filters. In [37], we showed that convolving images with non-separable filter banks learned by solving the problem of Eq. (1) and training an SVM on the output of those filters outperforms these other methods. Unfortunately, this requires many such non-separable filters, making it an impractical approach for large images or image stacks, whose usage is becoming standard practice in medical imaging. We show that our approach solves this issue.

\subsubsection{Pixel Classification}

In the $2 \mathrm{D}$ case we considered the two biomedical datasets of Fig. 6:

- The DRIVE dataset [41] is a set of 40 retinal scans captured for the diagnosis of various diseases. The dataset is split into 20 training images and 20 test images, with two different ground truth sets traced by two different human experts for the test images.

- The BF2D dataset is composed of minimum intensity projections of bright-field micrographs of neurons. The images have a very high resolution but exhibit a low signal-to-noise ratio, because of irregularities in the staining process. Furthermore, parts of the dendrites often appear as point-like structures that can be easily mistaken for the structured and unstructured noise affecting the images.

As mentioned above, we showed in [37] that the NON-SEP approach outperforms other recent approaches [16], [39] that rely on Machine Learning but is slow. Our goal is therefore to achieve the same level of performance but much faster. For completeness, we also compare our results to those obtained using the Optimally Oriented Flux [22], which we will refer to as $O O F$, which is widely acknowledged to be one of the best techniques for finding curvilinear structures using handdesigned filters.

In these experiments we replaced the SVM classifiers we used earlier [37] by Random Forests [8]. They not only brought a considerable speed improvement, but also resulted in better performance. Note that we do not need to compute the linear combination of the filter outputs in the case of $S E P$ $C O M B$ and $S E P-T D$, since the Random Forest classifier relies on linear projections. We will therefore opt for SEP-COMB* and $S E P-T D^{*}$.

We first learned a filter bank with 121 learned filters of size $21 \times 21$ on the DRIVE dataset and one on the BF2D dataset, as these parameters provided us with the best results. We have then learned other filter banks of reduced cardinality, both fullrank and separable, to assess the impact of the filter bank size on the final classification performance. 

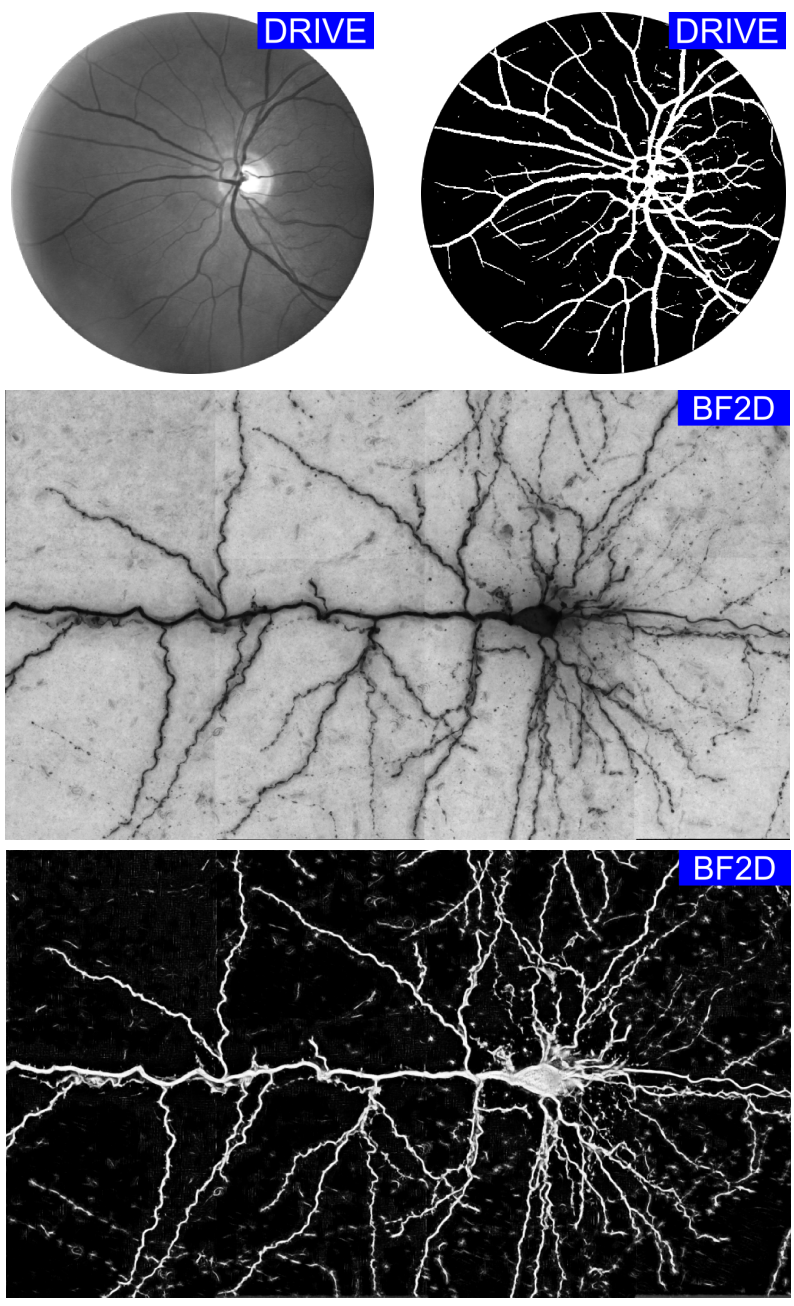

Fig. 6: Representative images from the $2 \mathrm{D}$ medical datasets considered, together with the corresponding pixel classification results obtained with our SEP-COMB* method.

As there is no universally accepted metric to evaluate pixel classification performance, we used several to compare our method to others. In particular, we considered the following:

- Area Under Curve (AUC): represents the area subtended by the ROC curve. It assumes values in $[0,1]$, the higher the better;

- F-measure [44]: assumes values in $[0,1]$, the higher, the better;

- Variation of Information (VI) [29]: assumes values in $[0, \infty)$

- Rand Index (RI) [43]: assumes values in $[0,1]$, the higher, the better.

The classification performance is tabulated using these different metrics in Table 2. SEP-COMB* and SEP-TD* performs consistently best, closely matching the performance of $\mathrm{NON}$ $S E P$ but with a significant speed-up. SEP-DIRECT is just as fast but entails a loss of accuracy. Somewhat surprisingly, $S E P$ $S V D$ falls between SEP-DIRECT and SEP-COMB* in terms of accuracy but is much slower than both. Finally, NON-SEP$F F T$ yields exactly the same results as $N O N-S E P$ as it should be, but it is much slower than plain $2 \mathrm{D}$ convolutions. The costs of the Fourier Transform are indeed amortized only for
TABLE 2: Analytic measure of the performance of the pixel classification task over different datasets. For the DRIVE dataset two ground truth are provided. We use the first one as reference and compute the analytic measure for the second one. For the other methods, the VI and RI values are compared on the classification thresholded at the value found using the F-measure. The values are averaged over 5 random trials and over the whole dataset images. For the learning-based approaches, a training set of 50000 positive and 50000 negative samples and a Random Forests classifier have been used. Approaches that use a separable filter basis have been found to reduce the computational costs by a factor of 10 in classifications tasks.

\begin{tabular}{lccccc}
\hline Method & AUC & F-measure & VI & RI & Time[s] \\
\hline Human Expert \#2 & - & 0.788 & 0.380 & 0.930 & - \\
OOF & 0.933 & 0.695 & 0.569 & 0.770 & 5.70 \\
NON-SEP(121) & 0.959 & 0.782 & 0.554 & 0.890 & 2.22 \\
NON-SEP-FFT(121) & 0.959 & 0.782 & 0.554 & 0.890 & 4.79 \\
SEP-SVD $(121)$ & 0.955 & 0.773 & 0.563 & 0.887 & 1.02 \\
SEP-DIRECT(25) & 0.948 & 0.756 & 0.602 & 0.879 & 0.23 \\
SEP-COMB*(25) & 0.959 & 0.785 & 0.541 & 0.894 & 0.23 \\
SEP-TD*(25) & 0.962 & 0.786 & 0.572 & 0.888 & 0.23 \\
\hline & & BF2D & & & \\
\hline OOF & 0.958 & 0.677 & 0.325 & 0.891 & 15.88 \\
NON-SEP(121) & 0.983 & 0.754 & 0.300 & 0.945 & 11.42 \\
NON-SEP-FFT(121) & 0.983 & 0.754 & 0.300 & 0.945 & 23.04 \\
SEP-SVD $(121)$ & 0.982 & 0.749 & 0.306 & 0.943 & 6.67 \\
SEP-DIRECT(25) & 0.980 & 0.750 & 0.306 & 0.944 & 1.44 \\
SEP-COMB*(25) & 0.981 & 0.752 & 0.301 & 0.944 & 1.44 \\
SEP-TD*(25) & 0.980 & 0.749 & 0.304 & 0.936 & 1.44 \\
\hline
\end{tabular}

extremely large image and filter sizes. Hence, it can be inferred that expressing the learned full-rank filter bank in terms of few separable filters leads to a significant speed-up at no cost in terms of accuracy.

The accuracy of $O O F$ is significantly lower than that of filtering-based approaches.

\subsubsection{Voxel Classification}

We also evaluated our method on classifying voxels as belonging or not to curvilinear structures in 3D volumes of Olfactory Projection Fibers (OPF) from the DIADEM challenge [2], which were captured by a confocal microscope. We first learned the non-separable 3D filter bank made of 49 $13 \times 13 \times 13$ pixel filters depicted by Fig. 11(a) using Eq. (1) and then the 16 separable filters. Figs. 3(d) and 11(b) show the separable filters learned using the $S E P-C O M B$ and $S E P-T D$ approaches respectively.

Separable filters are learned as described in Section 4. As in the $2 \mathrm{D}$ case, we then trained classifiers to use these filters, but we used $\ell_{1}$-regularized logistic regressors instead of Random Forests since they have proved faster without significant performance loss. For training we used a set of 200000 samples, randomly selected from 4 train images. Since these classifiers do not require us to compute the linear combination of the separable filter outputs, we chose again the $S E P-C O M B^{*}$ and $S E P-T D^{*}$ approach for our experiments.

We use NON-SEP as our baseline. We compare $S E P$ $C O M B^{*}$ and $S E P-T D^{*}$ against $N O N-S E P-F F T$, a $3 \mathrm{D}$ version of $O O F$ and $S E P-C P D$.

The results are essentially the same as in the $2 \mathrm{D}$-case. $S E P$ $C O M B^{*}$ and $S E P-T D^{*}$ are 30 times faster than NON-SEP-FFT 
TABLE 3: Analytic measure of the performance of the voxel classification task over the OPF dataset. The VI and RI values are compared on the classification thresholded at the value found using the F-measure. For the learning-based approaches, a training set of 200000 randomly selected samples and a $\ell_{1}$-regularized logistic regressor classifier have been used. Approaches that use a separable filter basis have been found to reduce the computational costs by a factor of 30 in classifications tasks.

\begin{tabular}{lccccc}
\hline Method & AUC & F-measure & VI & RI & Time[s] \\
\hline & \multicolumn{3}{c}{ OPF:Image 4 } \\
\hline OOF & 0.997 & 0.531 & 0.012 & 0.998 & 193.05 \\
NON-SEP-FFT(49) & 0.997 & 0.571 & 0.013 & 0.998 & 339.01 \\
SEP-CPD(49) & 0.997 & 0.567 & 0.013 & 0.998 & 40.06 \\
SEP-COMB*(16) & 0.997 & 0.570 & 0.013 & 0.998 & 11.08 \\
SEP-TD*(16) & 0.997 & 0.567 & 0.013 & 0.998 & 11.08 \\
\hline
\end{tabular}

for virtually the same accuracy. They are 4 times faster than $S E P-C P D$. As before, $O O F$ is even worse in terms of accuracy.

\subsection{Denoising}

To evaluate how good our approaches are at representing a set of generic filters in a very different context, we used them to approximate the 256 denoising filters computed by the KSVD algorithm [13], some of which are depicted by Fig. 7(b). We experimented with different sizes of the approximating separable filter bank, and reported the results in Table 4. As can be seen, the 36 separable filters shown in Fig. 7(a) are already enough to obtain a very accurate approximation, giving a perfect reconstruction of the original filters up to a nearly imperceptible smoothing of the filters with many highfrequency components.

Table 4 reports the denoising scores, measured using the Peak Signal-to-Noise Ratio (PSNR). [38] also considered the approximation of filter banks learned with the K-SVD algorithm by using sparse linear combinations of separable filters computed from a 1D DCT basis. However, we need significantly fewer separable filters, only 36 compared to the 100 required by [38].

Interestingly, the basis of separable filters we learn seem general. We proved that by taking the filters that were learned to approximate a filter bank of a specific image, and we used them to reconstruct the filter banks of the other images. In other words, we kept the same $\mathbf{s}^{k}$ filters learned for the Barbara image, and only optimized on the $w_{j}^{k}$ weights in Eq. (7) and Eq. (9). The results are summarized in Table 4.

\subsection{Convolutional Neural Networks}

In recent years, Convolutional Neural Networks (CNNs) have become increasingly popular and have been shown to improve upon the previous state-of-the-art for many challenging tasks. However, they are computationally intensive and this remains a barrier to their wide acceptance. In this section, we show that replacing the non-separable filters they typically use by separable ones can help alleviate this problem.

Recent works such as [11], [12] have addressed this issue, albeit in a different way. The authors of [11] focus on reducing complexity at training time. They show that thanks to the correlation present in the weights, it is possible to

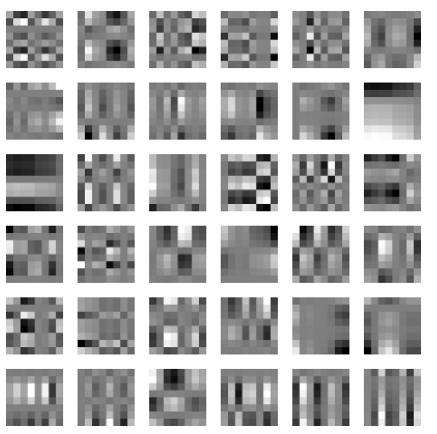

(a)

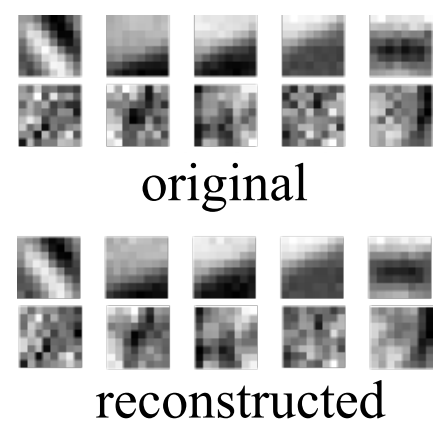

(b)
Fig. 7: Approximating an existing filter bank. (a) The 36 separable filters learned by $S E P-C O M B$ to approximate a bank of 256 filters learned with the K-SVD algorithm of [13]. (b) Comparison between some of the original filters learned by K-SVD (top row) and their approximations reconstructed by our algorithm (bottom row). While filters with a regular structure are very well approximated, noisy filters are slightly smoothed by the approximation. Their role in the denoising process is, however, marginal, and therefore this engenders no performance penalty.

TABLE 4: Results for the image denoising task. The denoising results are given in terms of Peak Signal-to-Noise ratio (PSNR) and measured in decibels. The images are corrupted by additive white gaussian noise with a standard deviation of 20 . The filters learned by K-SVD algorithm are approximated using different separable filter learning approaches. In the last part of the experiment, the filters learned from the Barbara image are used to denoise other images in order to assess how generic the denoising filters are.

\begin{tabular}{lccccc}
\hline & Barbara & Boat & House & Lena & Peppers \\
\hline Noisy image & 22.11 & 22.11 & 22.13 & 22.11 & 22.11 \\
\hline K-SVD $(256)$ & 30.87 & 30.39 & 33.36 & 32.40 & 32.34 \\
\hline SEP-COMB(25) & 30.10 & 30.30 & 33.04 & 32.37 & 32.08 \\
SEP-TD $(25)$ & 30.21 & 30.33 & 33.18 & 32.39 & 32.23 \\
SEP-COMB(36) & 30.76 & 30.38 & 33.23 & 32.39 & 32.27 \\
SEP-TD $(36)$ & 30.79 & 30.39 & 33.27 & 32.41 & 32.10 \\
SEP-COMB(49) & 30.86 & 30.40 & 33.30 & 32.40 & 32.30 \\
SEP-TD $(49)$ & 30.87 & 30.40 & 33.33 & 32.40 & 32.31 \\
SEP-COMB(64) & 30.87 & 30.39 & 33.35 & 32.40 & 32.33 \\
SEP-TD(64) & 30.87 & 30.39 & 33.35 & 32.40 & 32.33 \\
\hline SEP-COMB(36)-Barbara & - & 30.01 & 31.76 & 32.26 & 31.83 \\
SEP-TD(36)-Barbara & - & 30.02 & 31.71 & 32.27 & 31.82 \\
SEP-COMB(64)-Barbara & - & 30.04 & 31.97 & 32.27 & 31.93 \\
SEP-TD(64)-Barbara & - & 30.05 & 31.96 & 32.27 & 31.93 \\
\hline
\end{tabular}

optimize only a small fraction of the parameters and predict the remaining ones starting from them, without losing accuracy at test time.

The approach of [12] is more similar in spirit to ours. They reduce the complexity of the convolutional layers at test time by using smaller but non-separable operators, reducing computation by a 1.6 factor. Using separable filters learned with our method instead, we will show up to a factor 3 speed up. Moreover, the three approaches could be combined to achieve an even greater-speed up. A network could be trained using the approach of [11], then the decomposition [12] could be applied to obtain a smaller set of filters, to be turned into separable ones by using our approach.

In our experiments, we considered the following two datasets:

- The MNIST dataset [25] is a standard Machine Learning benchmark that consists of 70000 images of hand-written 
TABLE 5: Handwritten digit recognition on MNIST dataset with convolutional neural networks. Different kernel sizes are used in the first and second convolutional layers to evaluate the effect of kernel size on the classification performance and the execution time. The classification results and the execution times are reported for separable and non-separable filters. When separable filters are used in convolutions, the linear combinations of the convolutions of separable filters are computed in order to approximate the convolutions with non-separable filters. By using the separable filters, we can divide the computational times by 2 , at the cost of a negligible accuracy loss.

\begin{tabular}{cccccc}
\hline \multicolumn{6}{c}{ MNIST } \\
\hline \multicolumn{2}{c}{ Kernel Size } & \multicolumn{2}{c}{ Misclassification Rate } & \multicolumn{2}{c}{ Execution Time } \\
\hline $1^{\text {st }}$ Layer & $2^{\text {nd }}$ Layer & SEP & NONSEP & SEP & NONSEP \\
\hline 5 & 5 & $5.27 \%$ & $5.17 \%$ & 27.33 & 28.77 \\
5 & 9 & $4.84 \%$ & $4.17 \%$ & 24.9 & 44.61 \\
9 & 9 & $3.47 \%$ & $3.17 \%$ & 21.9 & 48.54 \\
\hline
\end{tabular}

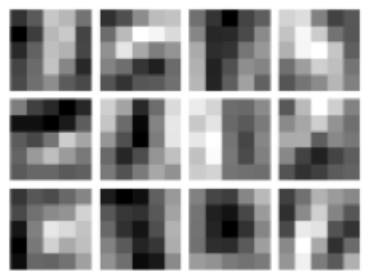

(a)

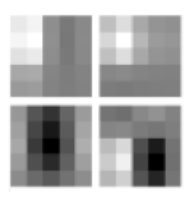

(b)

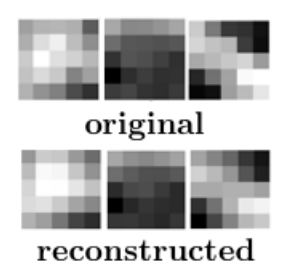

(c)
Fig. 8: Filters used in convolutional neural networks. (a) 12 filters going out of a node of the second convolutional layer. (b) A set of 4 separable filters obtained after tensor decomposition. (c) Comparison of the original and the approximated filters.

digits. The training set contains 60000 images and the test set 10000 . A batch size of 50 is used with a learning rate of 1 for 5 epochs to train the CNN.

- We built a Drone Detection dataset that consists of $40 \times$ 40 images in which a drone rotorcraft may or may not appear. The task is to say whether the drone is present or not, with a view to automated visual collision avoidance in swarms of such drones. The training and test datasets both contain 10950 images. Representative samples are shown in Fig. 9. Note that they are low-resolution and subject to motion-blur. A batch size of 10 is used with a learning rate of 1 for 100 epochs in training.

We consider an architecture consisting of 4 fully connected hidden layers for the MNIST dataset and 5 fully connected hidden layers for the Drone Detection dataset. We trained the networks using different kernel sizes in order to study the influence on the performance and the execution time. For our experiments, we used a publicly available Deep Learning Matlab toolbox [32].

The first layer consists of 6 feature maps connected to the single input layer via 6 kernels. The second layer is a 2-by2 downsampling layer. The third layer consists of 12 feature maps connected to the 6 downsampling layers via 72 kernels. The fourth layer is again a 2-by-2 downsampling layer.

For digit classification, the feature maps obtained at the last layer are concatenated into feature vectors and fed into the last layer, which has 10 output neurons in order to do multiway classification between 10 handwritten digit characters.
TABLE 6: Drone detection task with convolutional neural networks. As for the MNIST dataset, we considered different kernel sizes in the convolutional layers. Using separable filters the execution time can be reduced up to a factor of 3 without decreasing accuracy.

\begin{tabular}{|c|c|c|c|c|c|c|}
\hline \multicolumn{7}{|c|}{ Drone Detection } \\
\hline \multicolumn{3}{|c|}{ Kernel Size } & \multicolumn{2}{|c|}{ Misclassification Rate } & \multicolumn{2}{|c|}{ Execution Time } \\
\hline $1^{\text {st }}$ Layer & $2^{\text {nd }}$ Layer & $3^{\text {rd }}$ Layer & SEP & NONSEP & SEP & NONSEP \\
\hline 5 & 5 & 5 & $0.17 \%$ & $0.16 \%$ & 9.72 & 17.55 \\
\hline 5 & 5 & 9 & $0.09 \%$ & $0.08 \%$ & 19.69 & 44.33 \\
\hline 5 & 9 & 9 & $0.02 \%$ & $0.02 \%$ & 36.35 & 102.15 \\
\hline
\end{tabular}
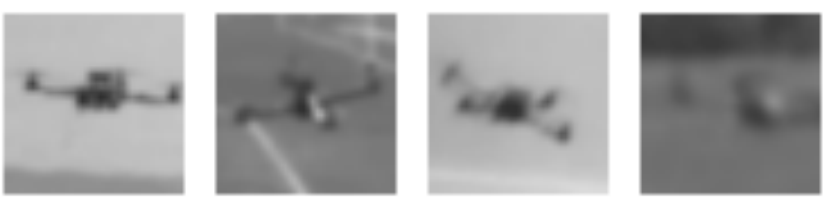

Fig. 9: Some training images from the Drone Detection dataset. A convolutional neural network is trained to classify images containing a rotorcraft drone. Separable filters can be used to speed up the execution time of the convolutional layers.

For drone detection, one more convolution layer consisting of 24 feature maps fully connected to the fourth layer is added. These feature maps are fed into 2 output neurons in order to discriminate whether the image contains a drone or not.

To obtain separable kernels, we apply the SEP-TD approach at each convolution layer. For the MNIST dataset, the 6 kernels in the first layer are approximated using 3 separable filters. In the second layer, we group the 12 filters corresponding to each outgoing feature map together and approximate them by 4 separable filters independently. For the Drone Detection dataset, 4 separable filters are used to approximate 6 filters in the first convolution layer. In the second convolution layer, 12 filters at each outgoing feature map are approximated with 5 filters independently. In the third convolution layer, the 24 filters at each outgoing node of the third convolution layer are approximated by 9 filters.

The execution times and the misclassification rates are reported in Tables 5 and 6 for different kernel sizes. Using separable filters speeds up the convolutional neural network without loss in accuracy. In particular, for a kernel size of 9 , classification becomes two to three times faster. Note that the purpose of these experiments is not necessarily to achieve state-of-the-art performance in a given task using CNNs, but to prove that our approach can be used on an arbitrary CNN to speed up convolutions without loss in accuracy, which is what it does.

Fig. 8 shows 12 of the filters in the second convolutional layer learned on the MNIST dataset and the 4 separable filters used to approximate them. Fig. 8(c) presents a visual comparison between the original and the reconstructed filters.

\subsection{Comparison between SEP-COMB and SEP-TD}

In the previous sections we showed that an arbitrary filter bank can be approximated by linear combinations of separable filters. We also proved that such decomposition can be used in 
several Computer Vision tasks to decrease the computational complexity without substantial changes in accuracy.

In this section we compare $S E P-C O M B$ and $S E P-T D$ in terms of approximation error and learning time. In particular we will see that $S E P-T D$ has the following advantages compared to $S E P-C O M B$ :

- Parameter reduction: The only parameter of $S E P-T D$ is the number of separable filters used to approximate the original one, while $S E P-C O M B$ relies on a regularization parameter.

- Faster convergence: $S E P-T D$ approach converges faster than $S E P-C O M B$, as can be seen in Fig. 12. The advantage of using SEP-TD rather than SEP-COMB is more pronounced in the 3D case.

- Lower approximation error: This can be explained by the fact that in the SEP-TD approach, a non-separable filter is explicitly written as the sum of the products of 1D filters. This approach provides a better approximation quality then $S E P-C O M B$, which relies on a soft constraint to make the filter ranks low, as shown in Fig. 12 and Fig. 13. The visual quality of the approximation of a nonseparable filter bank by linear combinations of separable filters obtained with SEP-TD is illustrated in Figs. 10 and 11 .

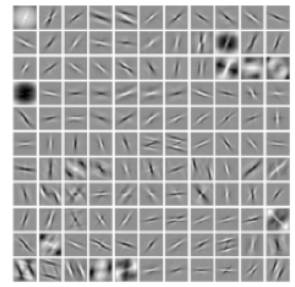

(a)

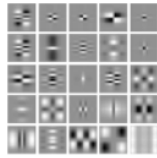

(b)

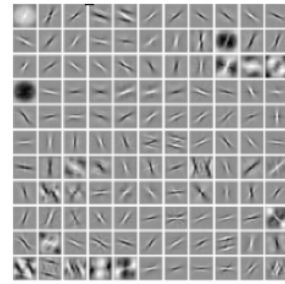

(c)
Fig. 10: Convolutional filter banks for classification in 2D. (a) Learned non-separable filter bank from DRIVE dataset, (b) separable filter bank learned with the SEP-TD approach, (c) reconstructed filter bank. The non-separable filters can be approximated accurately using a smaller set of separable filters.

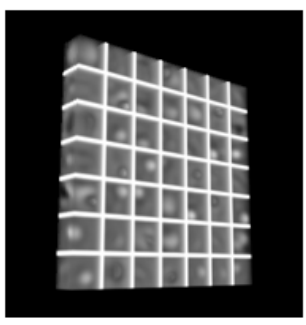

(a)

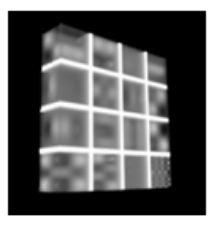

(b)

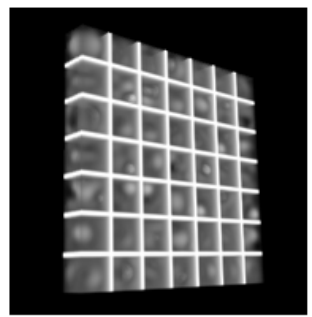

(c)
Fig. 11: Convolutional filter banks for classification in 3D. (a) Learned non-separable filter bank from OPF dataset, (b) separable filter bank learned with the $S E P-T D$ approach, (c) reconstructed filter bank. As in the 2D case, the non-separable filters can be approximated accurately using a smaller set of separable filters.

\section{Conclusion}

We have proposed a learning-based filtering scheme applied to the extraction of curvilinear structures, along with two learning-based strategies for obtaining separable filter banks. The first one directly learns separable filters by modifying the regular objective function. The second one learns a basis of separable filters to approximate an existing filter bank, and not only gets the same performance of the original, but also considerably reduces the number of filters, and thus convolutions, required. We presented two optimization schemes for this second approach. In the first one the separable filters are learned by lowering their ranks. In the second one, which proved to be more efficient and accurate, the filters are obtained by tensor decomposition.

Our techniques bring to learning approaches one of the most coveted properties of handcrafted filters, namely separability, and therefore reduce the computational burden traditionally associated with them. Moreover, designers of handcrafted filter banks do not have to restrict themselves to separable filters anymore: they can freely choose filters for the application at hand, and approximate them using few separable filters with our approach.

\section{REFERENCES}

[1] E. Acar, D. M. Dunlavy, and T. G. Kolda. A Scalable Optimization Approach for Fitting Canonical Tensor Decompositions. Journal of Chemometrics, 2011.

[2] G. Ascoli, K. Svoboda, and Y. Liu. Digital Reconstruction of Axonal and Dendritic Morphology DIADEM Challenge, 2010.

[3] F. Bach, R. Jenatton, J. Mairal, and G. Obozienski. Optimization with Sparsity-Inducing Penalties. Technical report, INRIA, 2011.

[4] C. Bauckhage. Tensor-based filter design using kernel ridge regression. In International Conference on Image Processing, 2007.

[5] C. Bauckhage, T. Käster, and J. K. Tsotsos. Applying ensembles of multilinear classifiers in the frequency domain. In Conference on Computer Vision and Pattern Recognition, 2006.

[6] Y. Bengio. Learning Deep Architectures for AI. Now Publishers, 2009.

[7] C. Bishop. Pattern Recognition and Machine Learning. Springer, 2006.

[8] L. Breiman. Random Forests. Machine Learning, 2001.

[9] H. Bristow, A. Eriksson, and S. Lucey. Fast Convolutional Sparse Coding. In Conference on Computer Vision and Pattern Recognition, 2013.

[10] A. Coates and A. Ng. The Importance of Encoding Versus Training with Sparse Coding and Vector Quantization. In International Conference on Machine Learning, 2011.

[11] M. Denil, B. Shakibi, L. Dinh, M. Ranzato, and N. de Freitas. Predicting parameters in deep learning. In Advances in Neural Information Processing Systems, pages 2148-2156, 2013.

[12] E. Denton, W. Zaremba, J. Bruna, Y. LeCun, and R. Fergus. Exploiting linear structure within convolutional networks for efficient evaluation. CoRR, abs/1404.0736, 2014.

[13] M. Elad and M. Aharon. Image Denoising via Sparse and Redundant Representations over Learned Dictionaries. IEEE Transactions on Image Processing, 2006.

[14] C. Farabet, B. Martini, P. Akselrod, S. Talay, Y. LeCun, and E. Culurciello. Hardware Accelerated Convolutional Neural Networks for Synthetic Vision Systems. In International Symposium on Circuits and Systems, 2010.

[15] M. Fazel, H. Hindi, and S. Boyd. A Rank Minimization Heuristic with Application to Minimum Order System Approximation. In American Control Conference, 2001.

[16] G. Gonzalez, F. Fleuret, and P. Fua. Learning Rotational Features for Filament Detection. In Conference on Computer Vision and Pattern Recognition, pages 1582-1589, 2009.

[17] S. Hawe, M. Seibert, and M. Kleinsteuber. Separable Dictionary Learning. In Conference on Computer Vision and Pattern Recognition, 2013.

[18] G. Hinton. Learning to Represent Visual Input. Philosophical Transactions of the Royal Society, 2010.

[19] K. Kavukcuoglu, P. Sermanet, Y.-L. Boureau, K. Gregor, M. Mathieu, and Y. LeCun. Learning Convolutional Feature Hierarchies for Visual Recognition. In Advances in Neural Information Processing Systems, 2010 . 


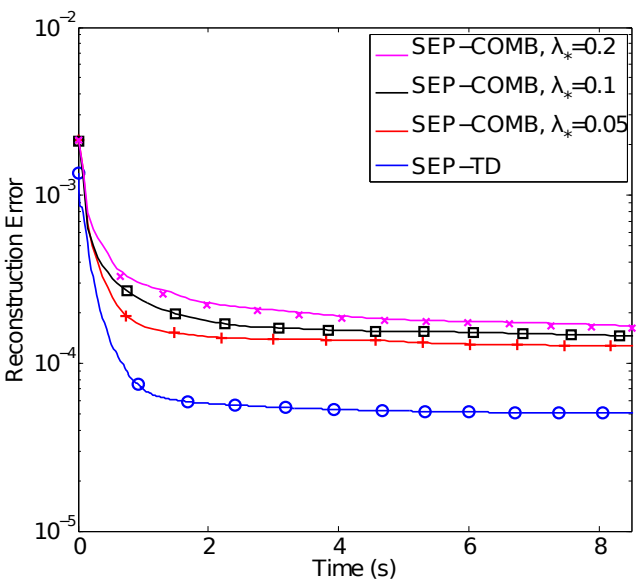

(a)

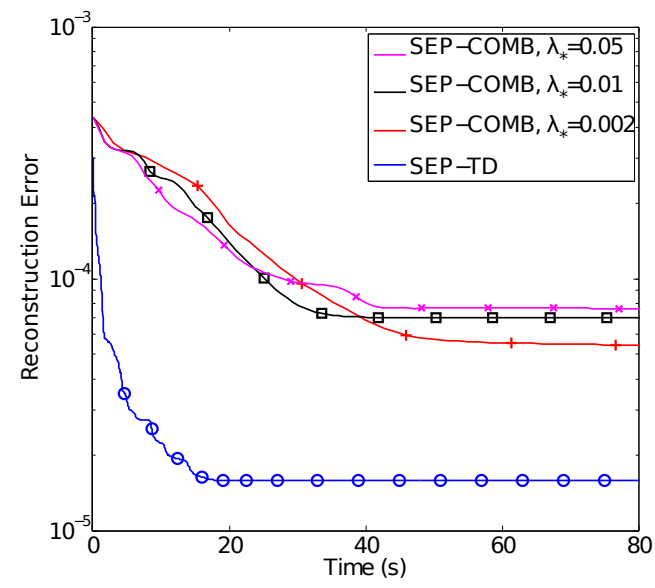

(b)

Fig. 12: Comparison of the reconstruction errors of $S E P-T D$ and $S E P-C O M B$ as a function of the learning time for approximating (a) a 2D non-separable filter bank and (b) a 3D non-separable filter bank. The performance of the SEP-COMB approach depends on the specified regularization parameter $\lambda_{*}$. Small regularization parameters yield a smaller reconstruction error. SEP-TD does not need to satisfy an additional constraint and yields a smaller reconstruction error compared to $S E P-C O M B$ with a faster convergence. In the $3 \mathrm{D}$ case, the difference is even more pronounced.

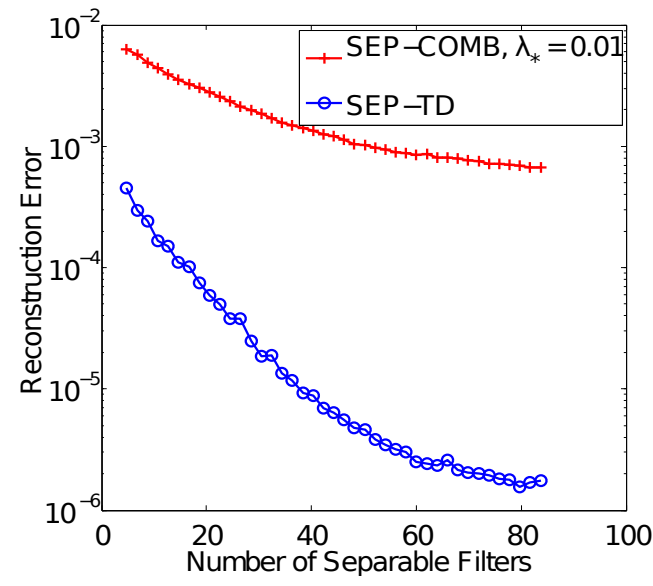

(a)

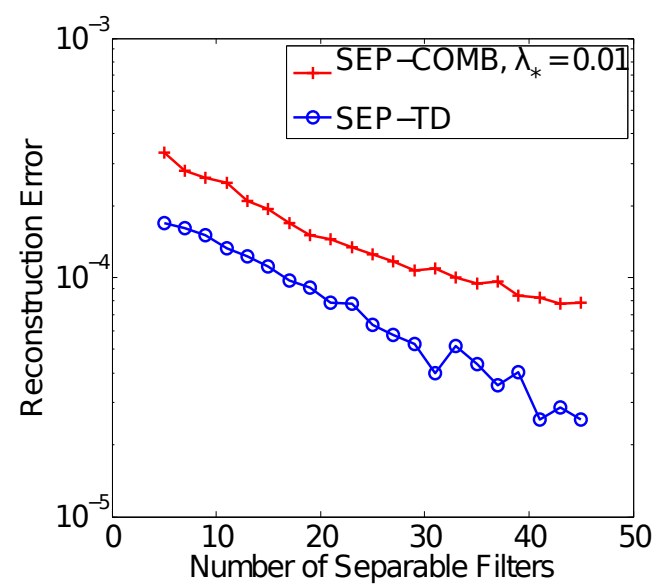

(b)

Fig. 13: Comparison of the reconstruction errors of $S E P-T D$ and $S E P-C O M B$ as a function of the number of separable filters used to approximate (a) a 2D non-separable filter bank of 121 filters and (b) a 3D non-separable filter bank 49 filters. The results are averaged over 10 repetitions. Also in this case we observe that $S E P-T D$ returns more accurate serults than $S E P-C O M B$ for every number of separable filters. Moreover, when the number of separable filters increase, the error decreases faster for SEP-TD than SEP-COMB.

[20] W. Kienzle, G. Bakir, M. Franz, and B. Schölkopf. Face detection - efficient and rank deficient. In Advances in Neural Information Processing Systems, pages 673-680, 2005.

[21] T. G. Kolda and B. W. Bader. Tensor Decompositions and Applications. SIAM Review, 2009.

[22] M. Law and A. Chung. Three Dimensional Curvilinear Structure Detection Using Optimally Oriented Flux. In European Conference on Computer Vision, 2008.

[23] Y. LeCun, L. Bottou, Y. Bengio, and P. Haffner. Gradient-Based Learning Applied to Document Recognition. Proceedings of the IEEE, 1998.

[24] Y. LeCun, L. Bottou, G. Orr, and K. Müller. Neural Networks: Tricks of the Trade, chapter Efficient Backprop. Springer, 1998.

[25] Y. LeCun and C. Cortes. The MNIST Database of Handwritten Digits.

$26]$ H. Lee, R. Grosse, R. Ranganath, and A. Ng. Convolutional Deep Belief Networks for Scalable Unsupervised Learning of Hierarchical Representations. In International Conference on Machine Learning, 2009.

[27] J. Mairal, F. Bach, J. Ponce, G. Sapiro, and A. Zisserman. Non-Local Sparse Models for Image Restoration. In International Conference on Computer Vision, 2009.

[28] F. Mamalet and C. Garcia. Simplifying Convnets for Fast Learning. In
International Conference on Artificial Neural Networks, 2012.

[29] M. Meilă. Comparing Clusterings - An Information Based Distance. JMVA, 2007.

[30] V. Mnih and G. Hinton. Learning to Detect Roads in High-Resolution Aerial Images. In European Conference on Computer Vision, 2010.

[31] B. Olshausen and D. Field. Sparse Coding with an Overcomplete Basis Set: A Strategy Employed by V1? Vision Research, 1997.

[32] R. B. Palm. Prediction as a Candidate for Learning Deep Hierarchical Models of Data. Master's thesis, Technical University of Denmark, 2012.

[33] P. Perona. Deformable Kernels for Early Vision. IEEE Transactions on Pattern Analysis and Machine Intelligence, 1995.

[34] H. Pirsiavash and D. Ramanan. Steerable Part Models. In Conference on Computer Vision and Pattern Recognition, 2012.

[35] R. Rigamonti, M. Brown, and V. Lepetit. Are Sparse Representations Really Relevant for Image Classification? In Conference on Computer Vision and Pattern Recognition, 2011.

[36] R. Rigamonti, A. Sironi, V. Lepetit, and P. Fua. Learning Separable Filters. In Conference on Computer Vision and Pattern Recognition, 2013.

[37] R. Rigamonti, E. Türetken, G. González, P. Fua, and V. Lepetit. Filter Learning for Linear Structure Segmentation. Technical report, EPFL, 2011.

[38] R. Rubinstein, M. Zibulevsky, and M. Elad. Double Sparsity: Learning 
Sparse Dictionaries for Sparse Signal Approximation. IEEE Transactions on Signal Processing, 2010.

[39] A. Santamaría-Pang, C. Colbert, P. Saggau, and I. Kakadiaris. Automatic Centerline Extraction of Irregular Tubular Structures Using Probability Volumes from Multiphoton Imaging. In Conference on Medical Image Computing and Computer Assisted Intervention, 2007.

[40] B. Savas and L. Eldén. Handwritten digit classification using higher order singular value decomposition. Pattern Recognition, 40(3):9931003, 2007.

[41] J. Staal, M. Abramoff, M. Niemeijer, M. Viergever, and B. van Ginneken. Ridge Based Vessel Segmentation in Color Images of the Retina. IEEE Transactions on Medical Imaging, 2004.

[42] S. Treitel and J. Shanks. The Design of Multistage Separable Planar Filters. IEEE Transactions on Geoscience Electronics, 1971.

[43] R. Unnikrishnan, C. Pantofaru, and M. Hebert. Toward Objective Evaluation of Image Segmentation Algorithms. IEEE Transactions on Pattern Analysis and Machine Intelligence, 2007.

[44] C. van Rijsbergen. Foundation of Evaluation. Journal of Documentation, 1974.

[45] J. Wright, Y. Ma, J. Mairal, G. Sapiro, T. S. Huang, and S. Yan. Sparse Representation for Computer Vision and Pattern Recognition. Proc. IEEE, 2010

[46] M. Zeiler, D. Krishnan, G. Taylor, and R. Fergus. Deconvolutional Networks. In Conference on Computer Vision and Pattern Recognition, 2010.

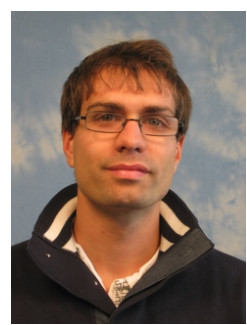

Amos Sironi joined EPFL in 2012 as Ph.D. candidate at CVLab, under the supervision of Prof. Pascal Fua and Prof. Vincent Lepetit. He received his B.Sc. in Mathematics from Università degli Studi di Milano-Bicocca and his M.Sc. in Mathematics from Univesità degli Studi di Padova. His main research interests include segmentation in medical images, machine learning and image descriptors.

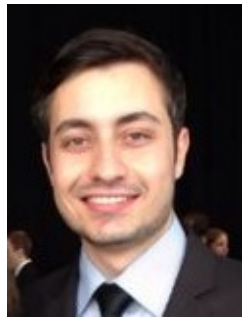

Bugra Tekin is pursuing his Ph.D. at CVLab in the field of human pose estimation and action recognition under the supervision of Prof. Pascal Fua. He received his M.Sc. degree in Electrical Engineering from Ecole Polytechnique Fédérale de Lausanne in 2013, and B.Sc degree in Electrical and Electronics Engineering from Bogazici University (Istanbul, Turkey) in 2011 with high honors. His research interests include 3D human pose estimation, action recognition and machine learning.

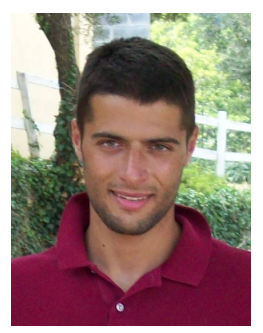

Roberto Rigamonti received his Ph.D. at the Computer Vision Laboratory, Ecole Polytechnique Fédérale de Lausanne, Switzerland in 2013. He received his BSc and MSc degrees in Computer Engineering from Politecnico di Milano, Italy, in 2007 and 2009 respectively. His main research interests include image descriptor learning, sparse coding, convex optimization, and machine learning.

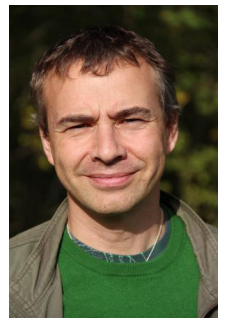

Vincent Lepetit is a Professor at the Institute for Computer Graphics and Vision, TU Graz and a Visiting Professor at the Computer Vision Laboratory, EPFL. He received the engineering and master degrees in Computer Science from the ESIAL in 1996. He received the PhD degree in Computer Vision in 2001 from the University of Nancy, France, after working in the ISA INRIA team. He then joined the Virtual Reality Lab at EPFL as a post-doctoral fellow and became a founding member of the Computer Vision Laboratory. His research interests include vision-based Augmented Reality, 3D camera tracking, Machine Learning, object recognition, and 3D reconstruction.

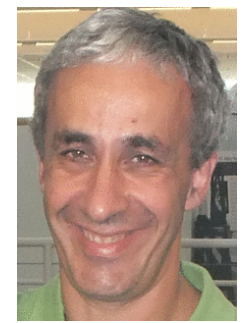

Pascal Fua received an engineering degree from Ecole Polytechnique, Paris, in 1984 and the Ph.D. degree in Computer Science from the University of Orsay in 1989. He joined EPFL in 1996 where he is now a Professor in the School of Computer and Communication Science. Before that, he worked at SRI International and at INRIA Sophia-Antipolis as a Computer Scientist. His research interests include shape modeling and motion recovery from images, analysis of microscopy images, and Augmented Reality. He has (co)authored over 150 publications in refereed journals and conferences. He is an IEEE fellow and has been a PAMI associate editor. He often serves as program committee member, area chair, or program chair of major vision conferences. 